\title{
Mineração
}

\section{Aplicação de prensas de rolos em minério de ferro}

\author{
(High pressure grinding rolls for iron ore)
}

\section{Farley Santos Ribeiro \\ Engenheiro de Minas, Anglo Ferrous Brazil E-mail: farley.ribeiro@angloferrous.com.br}

José Francisco Cabello Russo

Especialista de Processos, Anglo Ferrous Brazil E-mail: jose.russo@angloferrous.com.br

\section{Thiago Costa \\ Engenheiro Mecânico, Anglo Ferrous Brazil E-mail: thiago.costa@angloferrous.com.br}

\section{Resumo}

O Projeto Minas-Rio, de propriedade da Anglo Ferrous Brazil, prevê a utilização de prensa de rolos para cominuição do undersize do peneiramento (-25,4 mm). Esta é uma aplicação que se torna cada dia mais comum, porém relativamente nova em minério de ferro.

Foram realizados estudos no Centro de Pesquisa da Krupp/Polysius na Alemanha, com o objetivo de validar a aplicação de prensa de rolos para minério de ferro, de determinar os principais parâmetros usados no dimensionamento e de avaliar o impacto do aumento da umidade da alimentação na capacidade específica e na distribuição granulométrica do produto.

Os excelentes resultados obtidos, nos ensaios em escala-piloto, somados aos ganhos financeiros da aquisição e operação, à maior estabilidade do processo diante variações de $\mathrm{w}_{\mathrm{i}}$ (work index) e à distribuição granulométrica da alimentação, contribuíram para aprovar, de forma irrestrita, a aplicação de prensa de rolos para cominuição de minério de ferro no Projeto Minas-Rio.

Palavras-chave: Minério de ferro, prensa de rolos.

\section{Abstract}

The Minas-Rio Project that is being implemented by Anglo Ferrous Brazil, foresees the use of HPGR (High Pressure Grinding Rolls) to grind the undersize from screening at (-25.4 $\mathrm{mm})$. This application is recent for iron ore comminuting.

The study described herein, took place at the Krupp/Polysius Research Center, in Germany, aiming to validate the HPGR application for iron ore, as well as to determine the main parameters used in equipment sizing. The study also aimed to assesses the impact of moisture variation on ore feed in respect to specific grinding capacity and product size distribution.

The great results obtained from the sample tests that demonstrated more process stability for the work index $\left(w_{i}\right)$ and granulometric feed distribution variations, together with the possible financial gains from the HPGR implementation, contributed to the unrestricted approval of the HPGR application for iron ore comminuting in the Minas-Rio Project.

Keywords: Iron ore, high pressure grinding rollers. 


\section{Projeto Minas-Rio}

O Projeto Minas-Rio, de propriedade da Anglo Ferrous Brazil, está localizado nos estados de Minas Gerais e Rio de Janeiro. Trata-se de um projeto para produção de Pellet Feed de alto teor de ferro a partir de itabiritos, sendo que suas operações incluirão uma mina a céu aberto, britagem primária e secundária, peneiramento, transportadores de correia de longa distância, prensagem, moagem, flotação, espessamento, disposição de rejeitos, mineroduto e filtragem.

A planta de beneficiamento será localizada nos municípios de Conceição do Mato Dentro e Alvorada de Minas, distante cerca de $150 \mathrm{~km}$ de Belo Horizonte. O Pellet Feed produzido será escoado até o Porto de Açu por um mineroduto de, aproximadamente, $525 \mathrm{~km}$.

Essa planta foi projetada para alimentar $6.496 \mathrm{t} / \mathrm{h}$, com um rendimento operacional de 92,0\%, o que permitirá processar 52,3 MTA (milhões de toneladas ano, base seca), gerando uma produção de 24,5 MTA de concentrado final (Pellet Feed).

\section{Introdução}

Esse trabalho apresenta os principais fatores que levaram à utilização de prensa de rolos para redução de itabirito abaixo de 25 mm (após estágios de britagem primária e secundária), de forma agerar um produto prensado com $\mathrm{P}_{80}=1,0 \mathrm{~mm}$ (abertura na qual 80\% do material é passante), que irá alimentar a etapa moagem com aplicação de moinhos de bolas, gerando um produto com $\mathrm{P}_{80}=0,117 \mathrm{~mm}$ para posterior deslamagem e concentração por flotação.

A utilização de prensas de rolos ,na indústria, teve início em meados da década de 80, em aplicações de cominuição, na indústria de cimento, tratando material de britagem relativamente fácil. A partir de então, as prensas de rolos têm sido aplicadas com sucesso a materiais mais resistentes e abrasivos. Hoje, em minério de ferro, são utilizadas para britagem grosseira, britagem de pebbles de moinhos autógenos, remoagem prépelotização e briquetagem. A Mina de Los Colorados, de propriedade da Companhia Minera Del Pacifico, localizada no Chile, é um exemplo na utilização de prensas de rolos para granulometrias grosseiras em minério de ferro.

As prensas de rolos (HPGR, High Pressure Griding Rolls) são equipamentos de cominuição que consistem em um par de rolos girando em sentidos opostos, montados em um quadro rígido. Um rolo gira sobre eixo fixo no quadro, enquanto o outro eixo se desloca sobre guias, sendo posicionado por cilindros hidráulicos. A alimentação é introduzida na abertura entre rolos, onde a diminuição de tamanho ocorre pelo efeito da cominuição interparticular.

Em geral, a motivação para a utilização de prensas de rolos está relacionada à sua maior eficiência energética, se comparada com a eficiência dos britadores e dos moinhos convencionais, pois, nas prensas de rolos ocorre lenta aplicação de carga sobre as partículas, causando colapso estrutural dos grãos, de modo que a energia perdida em calor e ruído é minimizada.

Para o Projeto Minas-Rio foram compradas 03 prensas de rolos da Krupp/ Polysius, de 2,40 m de diâmetro e 1,65 m de largura, e capacidade de $2.200 \mathrm{t} / \mathrm{h}$ por equipamento. A potência instalada por equipamento será de $4.800 \mathrm{~kW}$, sendo 02 motores de $2.400 \mathrm{~kW}$ cada.

Há, atualmente, três fornecedores de prensas de rolos: Krupp/Polysius, KHD Humboldt Wedag AG e Koppern, todas sediadas na Alemanha.

\section{Objetivos}

Os principais objetivos desse estudo foram:

- Validar a aplicação das prensas de rolos ao tipo de minério.

- Obter os parâmetros requeridos para o dimensionamento (capacidade específica, pressão de moagem específica e energia necessária).
- Avaliar o impacto do aumento do teor de umidade na produtividade das prensas de rolos.

- Determinar a distribuição granulométrica do produto.

- Determinar a abrasividade do minério.

\section{Definições}

Nesse item, serão conceituados alguns termos usados especificamente em testes de prensas de rolos.

Capacidade específica - $\mathrm{t}^{*} \mathrm{~s} /$ $\left(m^{3 *} h\right):$ é definida como a produção horária (t/h) de cada máquina, dividida pela área projetada $\left(\mathrm{m}^{2}\right)$ e a velocidade periférica dos rolos (m/s).

“Força” de moagem ou pressão específica: é definida como a força hidráulica total $(\mathrm{kN})$ exercida nos rolos dividida pela área total dos rolos $\left(\mathrm{m}^{2}\right)$.

Consumo específico de energia: é definida como a quantidade de energia absorvida por tonelada de material alimentado.

\section{Metodologia}

Para validação da aplicação das prensas de rolos no Projeto Minas-Rio foram realizados dois testes-pilotos no Centro de Pesquisas da Krupp/Polysius na Alemanha. Foram processadas amostras provenientes da Serra do Sapo.

O equipamento utilizado, nos testes-piloto, foi uma prensa de rolos semi-industrial com as seguintes características:

- Diâmetro dos Rolos: 0,71 m.

- Largura dos rolos: 0,21 m.

- Velocidade dos rolos: 0,29 a 1,10 m/s.

- Tamanho máximo da alimentação: 16 a $35 \mathrm{~mm}$.

O primeiro teste foi realizado com $1.500 \mathrm{~kg}$ de amostra britada, cujo top size era de $25,4 \mathrm{~mm}$ e que continha mais de $60 \%$ de finos abaixo de 1,00 mm. O objetivo principal desse teste foi determinar qual a razão de redução poderia ser obtida com duas passadas do 
Farley Santos Ribeiro et al.

material nos rolos e qual o valor máximo de umidade a ser alimentado.

Já o segundo teste foi realizado com uma amostra de massa e top size semelhantes, porém um pouco mais fina que a enviada anteriormente. Com essa amostra foram realizados testes com uma única passagem na prensas e diferentes ajustes de pressão e teor de umidade.

Nesse artigo, serão discutidos apenas os resultados dos testes realizados com a segunda amostra enviada, cujo principal objetivo foi validar a aplicação das prensas de rolos no Projeto Minas-Rio.

\section{Resultados}

Todos os testes em escala-piloto foram realizados considerando uma velocidade constante dos rolos, variação da pressão de moagem de 1,8 a $3,8 \mathrm{~N} / \mathrm{mm}^{2}$, variação do teor de umidade de $6 \%$ a $9,0 \%$ na alimentação e uma única passagem na prensa de rolos. A Tabela 1 mostra os principais resultados dos testes.

\subsection{Capacidade específica}

Verifica-se na Figura 1, que a capacidade específica teve uma variação de 270 a $300 \mathrm{t*} /\left(\mathrm{h}^{*} \mathrm{~m}^{3}\right)$, para um teor de umidade entre $6,0 \%$ a $9,0 \%$, e uma pressão de moagem de 2,9 N/mm². Já aumentando a pressão de moagem para 3,9 N/mm², houve uma redução na capacidade específica de 10,0\% para uma amostra de 6,0\% de teor de umidade. Porém, reduzindo a força de moagem para 1,8 N/mm², a capacidade aumentou 10,0\%.

\subsection{Potência absorvida}

Alta pressão de moagem resulta em alta energia absorvida pelo material e, consequentemente, alto consumo específico de energia.

A potência absorvida pelo material, como mostrado na Figura 2, é encontrada como uma função linear da pressão de moagem e, nos testes realizados, teve uma variação entre 0,8 e 1,5 kWh/t para o intervalo de pressão de moagem testado.

\subsection{Distribuição granulométrica do produto}

As distribuições granulométricas da alimentação e dos produtos dos testes de prensagem foram realizadas através de peneiramento a seco. A amostra de descarga teve as porções laterais e central coletadas separadamente e suas frações foram analisadas individualmente.

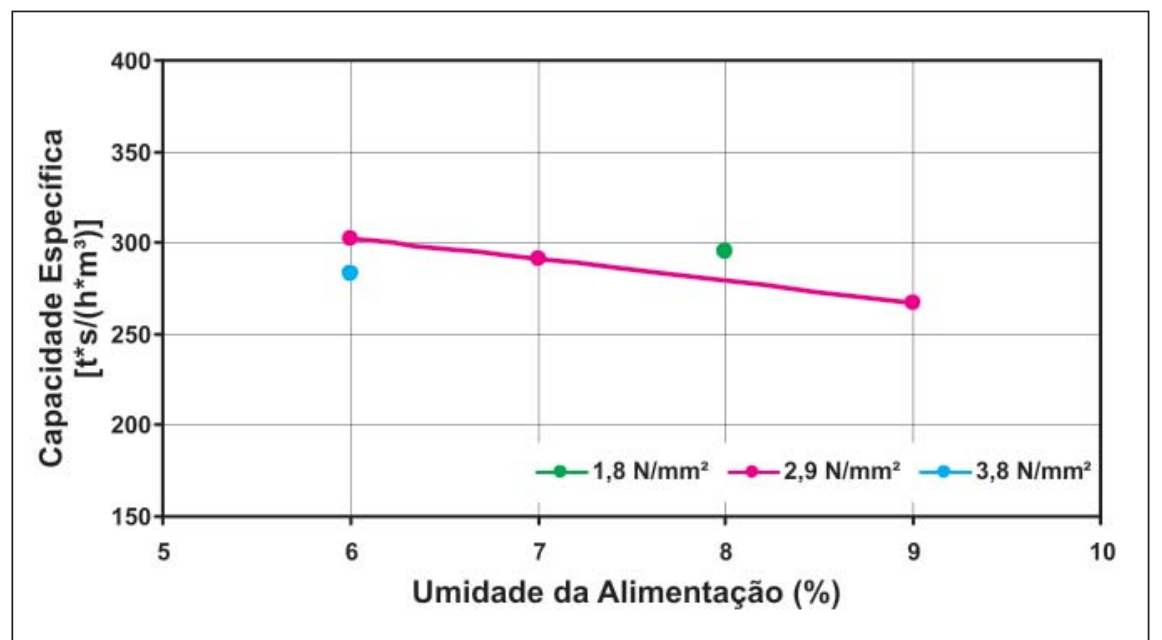

Figura 1 - Variação da capacidade específica em função do teor de umidade na alimentação.

Tabela 1 - Resultados dos testes em escala-piloto.

\begin{tabular}{|c|c|c|c|c|c|c|c|}
\hline \multirow{2}{*}{ Teste } & \multirow{2}{*}{$\begin{array}{c}\text { Umidade } \\
(\%)\end{array}$} & \multirow{2}{*}{$\begin{array}{l}\text { Capacidade } \\
\text { Específica } \\
\left(\mathbf{t}^{\star} \mathbf{s} /\left(\mathbf{h}^{\star} \mathbf{m}^{3}\right)\right)\end{array}$} & \multirow{2}{*}{$\begin{array}{c}\text { Pressão de } \\
\text { Moagem } \\
\text { Específica } \\
\left(\mathrm{Nmm}^{2}\right)\end{array}$} & \multirow{2}{*}{$\begin{array}{c}\text { Consumo } \\
\text { Específico } \\
\text { Energia }(k W h / t)\end{array}$} & \multicolumn{3}{|c|}{ Granulometria } \\
\hline & & & & & $\%<45 \mathrm{~mm}$ & $\%<90 \mathrm{~mm}$ & $\%<1,0 \mathrm{~mm}$ \\
\hline 1 & 6 & 302 & 2,93 & 1,13 & 40,1 & 62,2 & 87,6 \\
\hline 2 & 7 & 291 & 3,01 & 1,23 & 42,2 & 64,9 & 90,6 \\
\hline 4 & 9 & 267 & 2,89 & 1,18 & 41,9 & 64,6 & 90,2 \\
\hline 5 & 8 & 295 & 1,82 & 0,79 & 38,7 & 62,3 & 89,5 \\
\hline 6 & 6 & 283 & 3,75 & 1,51 & 42,7 & 64,9 & 90,4 \\
\hline
\end{tabular}


Aplicação de prensas de rolos em minério de ferro

As distribuições granulométricas da amostra global (descarga central + descarga lateral) são mostradas na Figura 3, onde se verifica que o top size do produto foi menor que $16,0 \mathrm{~mm}$, fato este que significa a abertura de trabalho dos rolos.

\subsection{Pressão específica de moagem e granulometria do produto}

A granulometria do produto é controlada, com certos limites, pela pressão de moagem aplicada. A correlação entre a pressão de moagem e a granulometria do produto obtido na descarga total é mostrada na Figura 4.

\subsection{Teste de abrasividade}

O teste de abrasividade é usado para determinar a taxa de desgaste para diferentes tipos de minério em prensa de rolos. Para essa determinação, é utilizado um equipamento com dois rolos polidos, fabricados em NiHard IV, e uma amostra de $100 \mathrm{~kg}$ britada abaixo de 3,15 $\mathrm{mm}$. Antes e depois de cada teste, os rolos são pesados e a taxa específica de desgaste é obtida dividindo a perda peso dos rolos pela massa total processada.

A pressão de moagem, o consumo de energia e a capacidade específica de alimentação são medidos e, além disso, a pressão de moagem deve ser ajustada, se necessário.

Para determinação do índice de abrasividade, foram realizados dois testes: um com material seco e outro com teor de umidade de $3,0 \%$. O teor de umidade de 3,0\% foi o máximo alcançando sem que ocorresse o deslizamento dos rolos.

Os resultados mostrados na Tabela 2 indicam que o material tem uma abrasividade relativamente alta, quando comparado com outros minérios.

A taxa de desgaste, em escala industrial, é calculada levando em conta o diâmetro e a velocidade dos rolos, o tipo e o comprimento dos studs aplicados e as características do material

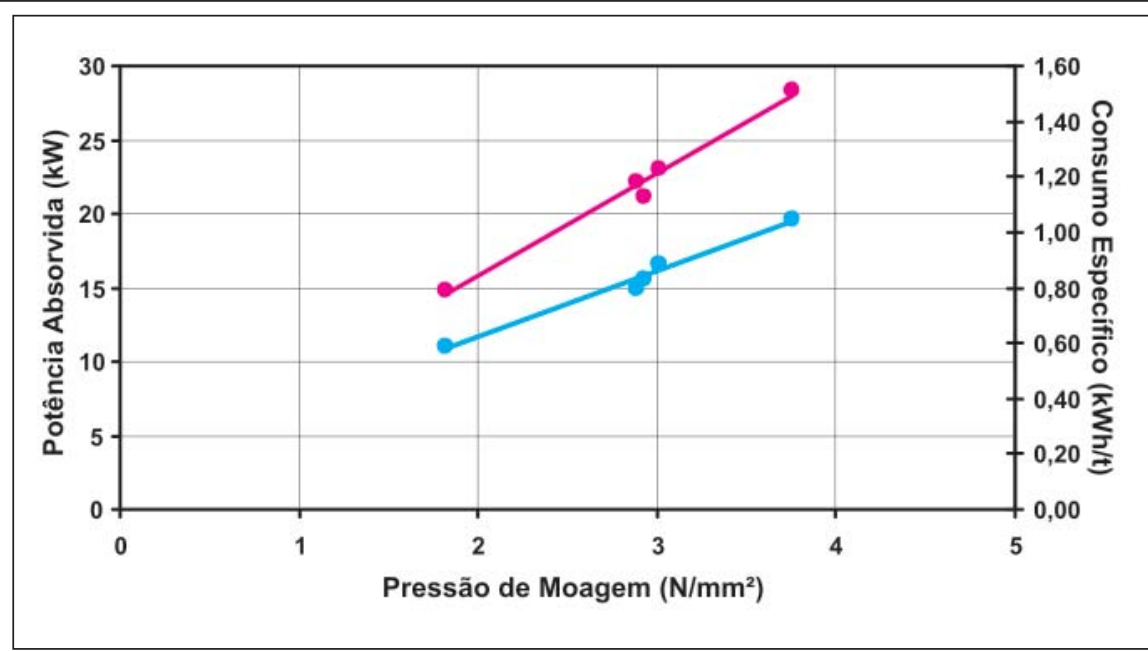

Figura 2 - Correlação entre pressão de moagem e consumo específico.

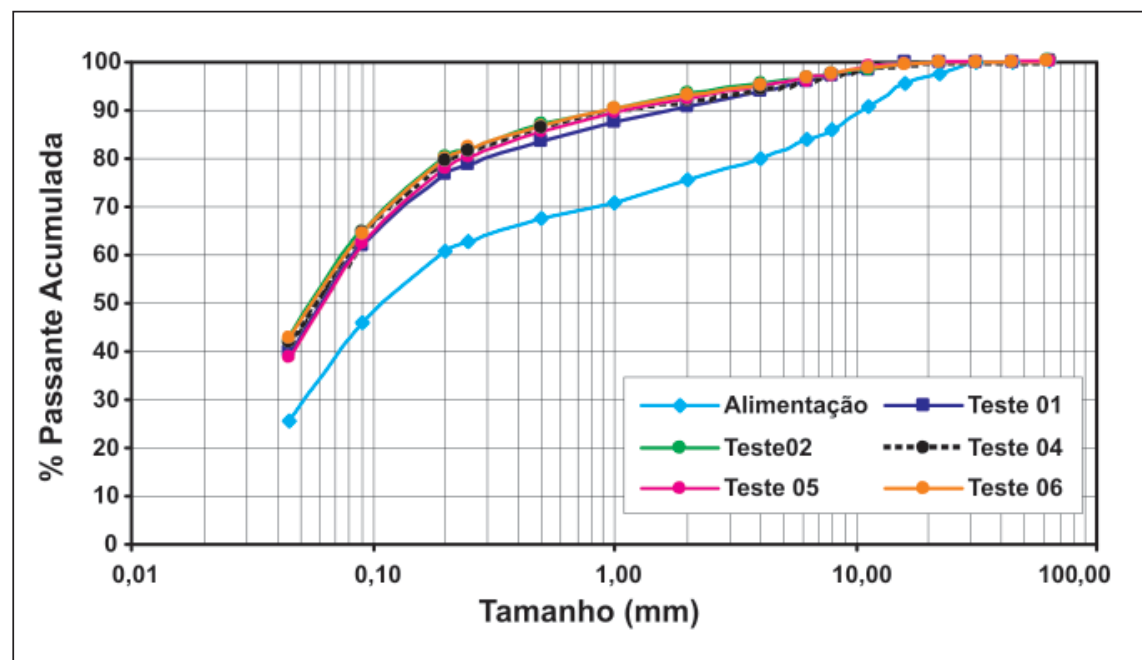

Figura 3 - Distribuição granulométrica do produto da prensa de rolos.

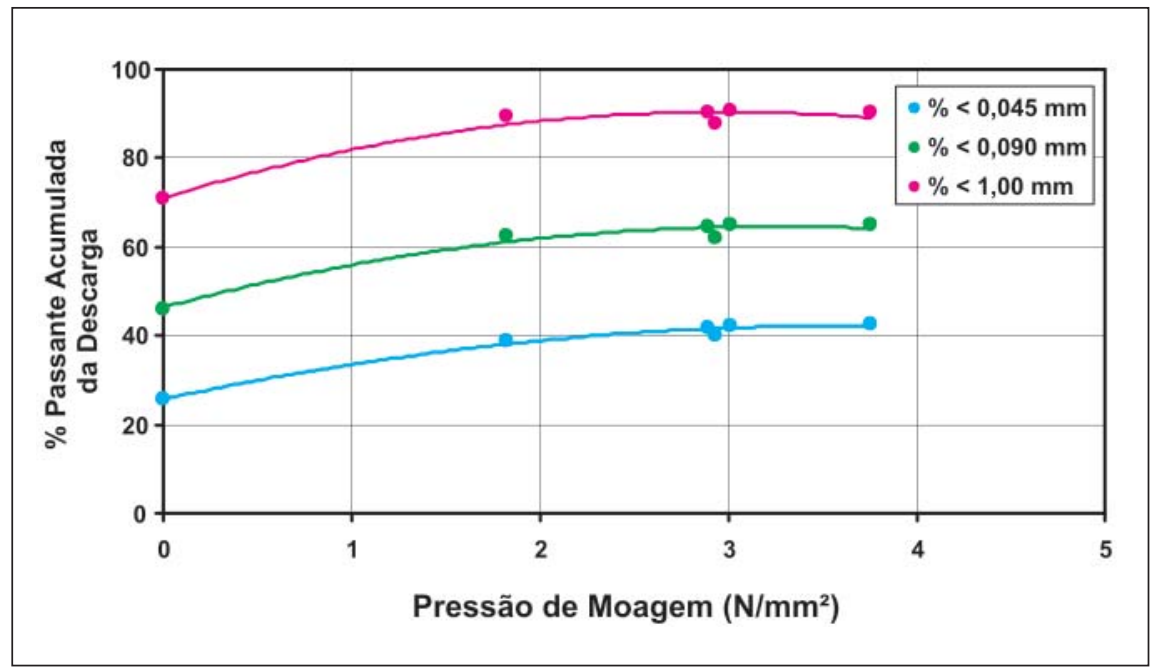

Figura 4 - Correlação da granulometria do produto em função da pressão de moagem. 
Farley Santos Ribeiro et al.

Tabela 2 - Resultados dos ensaios para determinação do índice abrasividade do material.

\begin{tabular}{c|c|c|c|c}
\hline Teste & Umidade (\%) & $\begin{array}{c}\text { Capacidade Específica } \\
\left(\mathbf{t}^{*} \mathbf{s} /\left(\mathbf{h}^{*} \mathbf{m}^{3}\right)\right.\end{array}$ & $\begin{array}{c}\text { Pressão de Moagem } \\
\text { Específica }\left(\mathbf{N} / \mathbf{m m}^{2}\right)\end{array}$ & $\begin{array}{c}\text { Taxa de Desgaste } \\
(\mathbf{g} / \mathbf{t})\end{array}$ \\
\hline 1 & 1,0 & 200,7 & 4,0 & 25,4 \\
\hline 2 & 3,0 & 142,3 & 4,0 & 31,6 \\
\hline
\end{tabular}

que será tratado, tais como a umidade e granulometria. Para o Projeto MinasRio, foi estimado que o revestimento dos rolos tenha uma vida útil estimada de 4.000 horas.

\section{Ganhos operacionais}

O objetivo do estudo de aplicação das prensas de rolos, no Projeto MinasRio, foi o de obter uma possível redução no consumo de energia, quando comparado com britadores cônicos e moinhos de bola convencionais. O processo de cominuição, na prensa de rolos, é, do ponto de vista energético, o mais eficiente, fato que ocorre em função da lenta aplicação de carga sobre as partículas, o que causa colapso estrutural dos grãos, minimizando o ruído e a energia perdida em forma de calor.

Durante o estudo de viabilidade foram comparadas duas propostas de circuitos. Na primeira delas, utiliza-se a prensa alimentada com o produto da britagem secundária e sua descarga alimenta a moagem com moinhos de bolas. Na segunda proposta, o minério proveniente da britagem secundária alimenta uma britagem terciária composta por britadores Barmac e seu produto alimenta a moagem de bolas, porém necessitaria de um número maior de moinhos.

O resultado do estudo mostrou que a utilização das prensas resultaria em uma potência instalada 15,0\% inferior ao circuito com britagem terciária. A necessidade de mão-de-obra, para operação e manutenção dos equipamentos, é 20,0\% maior, caso fosse utilizado britagem terciária. Outro fato relevante foi a redução no investimento dos equipamentos, ficando o preço da alternativa com prensas de rolos cerca de 6,0\% menor, também comparada com o circuito de britagem terciária. Portanto o fator decisivo, na utilização de um circuito com as prensas, é a redução no custo operacional.

Um ganho significativo, na manutenção, quando comparado aos moinhos e britadores, está relacionado à alta disponibilidade física. Tal fator, porém, depende da abrasividade do minério uma vez que o mesmo é diretamente proporcional ao número de trocas devido ao desgaste da superfície e das laterais dos rolos. Os revestimentos que utilizam o conceito de camada autógena de proteção, com pinos de carbeto de tungstênio, são mais adequados para aplicação em minérios abrasivos, o que resulta em melhores índices de disponibilidade e de tempo médio entre falhas do equipamento e em redução dos custos de manutenção da planta. O rolo montado utilizando o conceito de camada autógena (com os pinos) pode ser visto na Figura 5.
As prensas de rolos são sensíveis à segregação de material no chute de alimentação e à presença de corpos metálicos não desejáveis ao processo, que pode resultar em não prensagem do material e um desgaste excessivo dos rolos. Outro fator que deve ser considerado é a umidade do material alimentado, pois o desgaste dos rolos aumenta, significativamente, quando há um aumento da umidade na alimentação.

\section{Conclusão}

Com base nesse estudo, a aplicação de prensas de rolos, para cominuição de minério de ferro, foi aprovada, devido às seguintes vantagens:

- Reduziu o investimento inicial em, aproximadamente, 8,0\%, quando comparado com a aplicação de britagem terciária e peneiramento secundário.

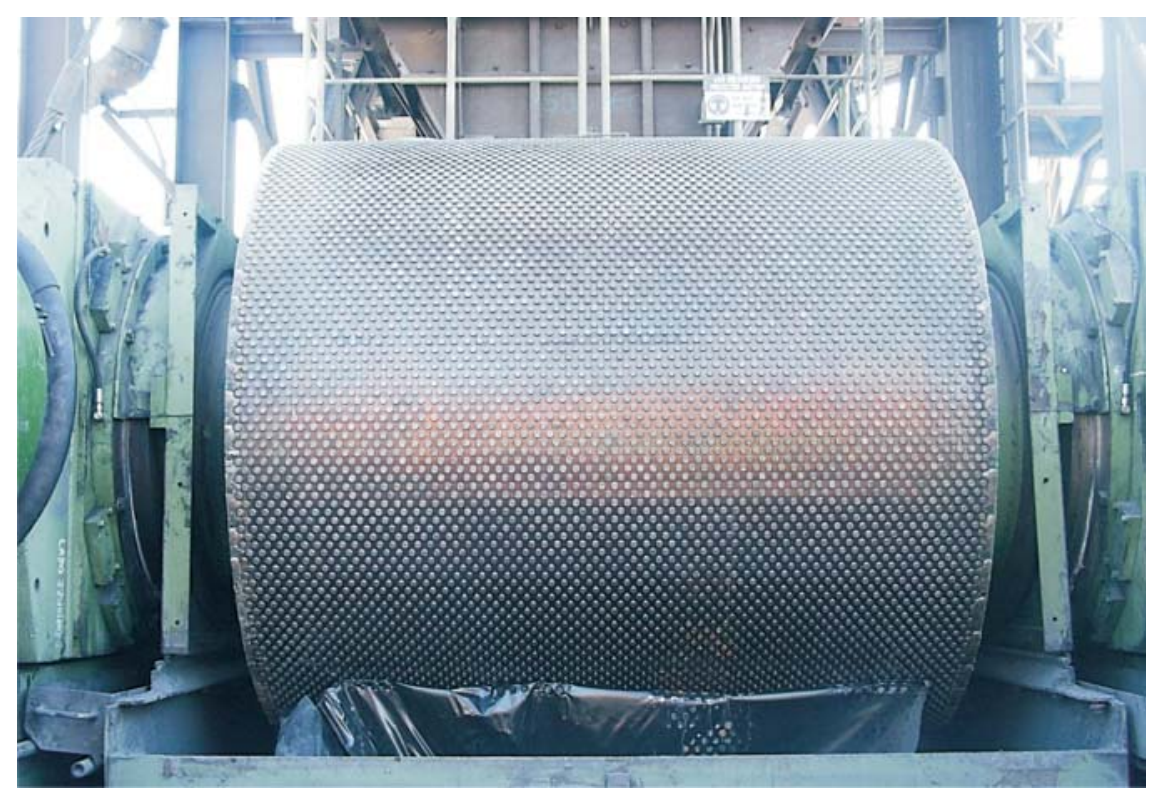

Figura 5 - Rolo montado com pinos de carbeto de tungstênio. 
- Reduziu, aproximadamente, 2,0\% no custo operacional, fato relacionado, principalmente, à maior eficiência enérgica das prensas de rolos.

- Permitiu a simplificação do circuito de cominuição da usina de beneficiamento, com eliminação dos circuitos de peneiramento secundário e britagem terciária.

- Irá gerar maior estabilidade no processo diante de uma variação do $w_{i}$ (work index) e da distribuição granulométrica do minério ao longo do desenvolvimento da mina, uma vez, que a capacidade específica aumenta com a dureza do minério.

- Reduziu a capacidade específica de, no máximo, $10,0 \%$, porque os teores de umidade foram de até 9,0\% na alimentação das prensas de rolos, nos ensaios de escala-piloto.

\section{Referências bibliográficas}

BURCHARDT, E. High Pressure Grinding Tests on Friable Itabirites from Serra do Sapo Mine. Polysius Research Centre. Alemanha: 2007.

ECM S.A - Projetos Industriais. Avaliação da utilização de prensa de rolos. Belo Horizonte: Projeto Básico do Sistema Minas-Rio, 2006.

GOOSSENS, M. Teste-piloto de concentração do minério de ferro da serra do Sapo. Belo Horizonte: Relatório Interno Sistema Minas-Rio, 2007.

KAWATRA, K. S. Advances in comminution. Society for Mining. Metallurgy and Exploration (SME). Estados Unidos da América: 2006.

KLYMOWSKY, R. High Pressure Grinding Tests on Coarse Iron Ore from Minas-

Rio System. Polysius Research Centre. Alemanha: 2007.

KLYMOWSKY, R., PATZELT, N., KNEEHT, J., BURCHARDT, E. Selection and

Sizing of High Pressure Grinding Rolls. Polysius Research Centre. Alemanha: 2005.

MULAR, A. L., HALBE, D. N., BARRAT, D. J. Mineral processing plant design.

Practice and Control Proceedings, v. 1, p. 636-667, 2002.

MORLEY, C. High pressure grinding rolls - a technology review. Society for Mining,

Metallurgy and Exploration (SME). Estados Unidos da América: 2006.

Artigo recebido em 30/07/2009 e aprovado em 11/01/2010.

\section{A REM tem novo endereço:}

\section{FUNDAÇÃO GORCEIX - REM}

Rua Carlos Walter Marinho Campos, 57 Bairro: Vila Itacolomy 35400-000 - Ouro Preto - MG $\begin{array}{ll}\text { (31) } 3551-4730 & \text { (31) 3559-7408 }\end{array}$

\section{www.rem.com.br}

